\title{
Evaluation of tear film and the morphological changes of meibomian glands in young Asian soft contact lens wearers and non-wearers
}

\author{
Tianpu Gu${ }^{1,2,3+}$, Lu Zhao ${ }^{1,2,3 \dagger}$, Zhuzhu Liu ${ }^{1,2,3}$, Shaozhen Zhao ${ }^{1,2,3}$, Hong Nian ${ }^{1,2,3^{*}}$ and Ruihua Wei ${ }^{1,2,3^{*}}$ (1)
}

\begin{abstract}
Background: The aim of this study was to explore the differences in terms of tear film and meibomian glands (MGs) between young Asian soft contact lens (CL) wearers and non-wearers.

Methods: A prospective, cross-sectional observational study was conducted using 148 subjects (63 non-wearers, and 85 soft CL wearers who had been wearing CLs for more than 1 year) recruited from a clinic in Tianjin, China. All subjects first responded to an Ocular Surface Disease Index (OSDI) questionnaire and then underwent a standardized dry eye examination, which included measuring tear meniscus height ( $(\mathrm{TMH})$, non-invasive tear breakup time (NIBUT), and corneal fluorescein staining (CFS). The MGs were evaluated via ImageJ, distorted MG count and the MG dropout were recorded.

Results: Compared to the control group (non-wearers), the $C L$ group recorded higher OSDI and CFS scores, lower TMH and NITBUT values, a larger distorted MG count, and larger MG dropout (all $P<0.05$ ). Pearson correlation analysis found a correlation between MG dropout and the duration of CL use $(r=0.440, P<0.001)$, OSDI $(r=0.298$, $P=0.006)$, and CFS scores $(r=0.442, P<0.001)$.

Conclusion: $\mathrm{CL}$ wearers showed higher MG dropout and reduced TMH and NITBUT, which likely contributes to severe CL-related dry eye symptoms. CL use may lead to a higher MG dropout rate, and the extent of the MG dropout presumably influences the tear film status in $\mathrm{CL}$ wearers.
\end{abstract}

Keywords: Contact lens, Meibomian glands, Meibomian gland dysfunction, Tear film young Asian

\section{Background}

There are an estimated 140 million people worldwide who wear contact lenses (CLs) for refractive error corrections [1]. During the past 20 years in China, there has been an enormous growth in the number of $\mathrm{CL}$ wearers, and the number of CL wearers is increasing every year [2].

Dry eye symptoms in CL wearers are more frequent and intense than those in non-wearers [3, 4]. Although lens designs and materials have advanced, a considerable number of soft CL wearers complain of clinically significant signs

\footnotetext{
* Correspondence: nianhong@126.com; rwei@tmu.edu.cn

${ }^{\dagger}$ Tianpu Gu and Lu Zhao contributed equally to this manuscript.

${ }^{1}$ Tianjin Key Laboratory of Retinal Functions and Disease, Tianjin 300384, China

${ }^{2}$ Eye Institute and School of Optometry, Tianjin 300384, China

Full list of author information is available at the end of the article
}

and symptoms, including foreign body sensations, redness, and CL-related dry eye [5-7]. About 30 to $50 \%$ of CL wearers report dry eye symptoms, and dry eye is gradually becoming a public health problem $[5,7,8]$. Moreover, the discomfort or irritation associated with dry eye may lead to intolerance of CLs [9-11]. Such symptoms should be addressed in time to prevent such an occurence [12].

As generally agreed upon, successful CLs wear depends on a stable tear film, including the production and maintenance of the constituents in each of the layers [11]. Several studies have shown that CLs produce a series of biochemical and biophysical changes in the ocular surface, such as in the integrity of the tear film and ocular surface microenvironment [13-15]. Ocular surface health cannot be sustained in the absence of a

(c) The Author(s). 2020 Open Access This article is distributed under the terms of the Creative Commons Attribution 4.0 International License (http://creativecommons.org/licenses/by/4.0/), which permits unrestricted use, distribution, and 
healthy lipid layer; hence, MG function has attracted the attention of many researchers around the world in recent decades [16]. However, little attention has been paid to the effect of CL on MGs in the Asian population, who tend to have absent or lower lid creases and more fat in the upper eyelid compared to the Caucasian population. Therefore, a better understanding of ethnicityrelated variations involving CL discomfort, especially between Asians and non-Asians, must be taken into consideration.

In this study, we use a novel, noncontact meibographic technique to evaluate the morphology of MGs to obtain a more precise calculation of the MG dropout rate in a young Asian population. The purpose of the current study is to explore the differences in the Ocular Surface Disease Index (OSDI), tear meniscus height (TMH), non-invasive tear breakup time (NIBUT), corneal fluorescein staining (CFS) scores, and MGs dropout between soft CL wearers and non-wearers. We also analyze the relationship between MG dropout and the other parameters, including the OSDI, TMH, NIBUT, CFS scores, and the duration of CL use.

\section{Methods}

The cross-sectional observational study was conducted at Tianjin University Eye Hospital (Tianjin, China) in June 2015. This study adhered to the tenets of the Declaration of Helsinki and was approved by the Institutional Ethical Committee Review Board of Tianjin University Eye Hospital. Written informed consent was obtained from all patients before their participation in the study.

All measurements were performed in an identical manner by the same professional operator, who was not aware of the CL wearing status of the subjects before the examinations. All measurements were performed from 9:00 AM to 11:00 AM to exclude the influence of diurnal variations. The ambient conditions of the examination room were kept constant, with the temperature varying from 24 to $28^{\circ} \mathrm{C}$ and the relative humidity varying from 40 to $50 \%$. Due to the similar nature of each individual's two eyes, only the right eye results were analyzed.

A previous study has shown that disruption to the precorneal tear film is short-lived and that the tear film returns mostly to normal within $25 \mathrm{~min}$ of CL removal [17]. After the CL was removed and more than $1 \mathrm{~h}$ later, the following examinations took place in sequence: TMH, NIBUT, MG dropout, the distorted MG count, and CFS.

\section{Subjects}

A total of 148 consecutive subjects enrolled in the optometry clinic were used in this study (Table 1), and these subjects were divided into the CL group (85 participants, $25.52 \pm 3.20$ years old, wearing CLs for more than 1 year) and the control group (63 nonwearers, $23.35 \pm 3.83$ years old).

The inclusion criteria for CL group were as follows: more than 18 years old and less than 30 years old; no history of any ocular diseases other than those associated with CL-related changes before wearing CLs; CL replacement based on a monthly or quarterly schedule with storage case replacement every 1 or 3 months; daily (5-7 d/wk., 5-12 h/d) wear, and not using any measures to manage dry eye symptoms, including warm compresses, artificial tear drops, and other medical eye drops. The control subjects were selected with the same age criteria, no contact lens wearing history, and no eye disease other than the dry eye. Exclusion criteria included patients who had active ocular infection or inflammation, recent ocular surgeries, or using medications that might affect the tear film.

\section{OSDI}

The OSDI questionnaire was utilized to quantify ocular discomfort, particularly in terms of measuring symptoms of discomfort related to dry eye. It has been validated and proven to be reliable [18]. The OSDI consists of 12 questions about symptoms of ocular discomfort and dryness. These questions are divided into three parts: vision-related symptoms (questions 1-5), ocular discomfort symptoms (6-9), and environmental stimuli (1012). Moreover, its total scores range from 0 to 100 , which indicate that the ocular surface is normal $(0-12$ points) or that the individual has mild (13-22 points), moderate (23-32 points), or severe (33-100 points) dry eye disease. All participants were instructed to finish the questionnaire.

\section{TMH}

Using the tear analysis software on the Keratograph $5 \mathrm{M}$ (Oculus, Wetzlar, Germany) [19], TMH was measured at the center of the lower tear meniscus with a graticule in $0.01 \mathrm{~mm}$ units. A mean value for the TMH was recorded after three successive measurements of at least $60 \mathrm{~s}$ in duration.

\section{NITBUT}

The NITBUT was measured using the Keratograph $5 \mathrm{M}$ with no fluorescein dye, as described in a previous study [20]. After the alignment of the instrument head with the center of the pupil, the participant was asked to blink three consecutive times and then instructed to hold his/her eyes open for as long as possible. The time elapsed between the last blink and the first sign of distortion occurring of the ring pattern was recorded as the NITBUT. The measurement was repeated three times for each eye, and the average was noted. 
Table 1 Comparison of the age, sex ratio, OSDI, TMH, NITBUT, and CFS scores for participants from both groups

\begin{tabular}{lllllll}
\hline & Age (years) & Sex Ratio (M/F) & OSDI (scale 0-100) & TMH (mm) & NIBUT (s) & CFS scores (scale 0-12) \\
\hline CL group & $25.52 \pm 3.20$ & $23 / 62$ & $17.21 \pm 8.67$ & $0.21 \pm 0.06$ & $6.95 \pm 4.79$ & $2.02 \pm 1.48$ \\
Control group & $23.35 \pm 3.83$ & $18 / 45$ & $11.41 \pm 6.00$ & $0.24 \pm 0.08$ & $9.26 \pm 2.95$ & $1.06 \pm 0.88$ \\
$P$ & 0.065 & 0.622 & $<0.001$ & 0.040 & $<0.001$ & $<0.001$
\end{tabular}

CL contact lens, OSDI the ocular surface disease index, TMH tear meniscus height, NIBUT noninvasive tear breakup time, CFS corneal fluorescein staining, $\mathrm{M} / \mathrm{F}$ male/female

\section{Meibography}

As described in a previous study [21], high-contrast meibography images of MGs were obtained using Keratograph $5 \mathrm{M}$ 's infrared camera system and then analyzed digitally using ImageJ (National Institute of Health; http://imagej.nih.gov/ij). Using a single-handed technique is convenient for the eversion of the upper and lower eyelids when obtaining the meibography images [22]. Using the freehand selection tool in ImageJ, the area of loss was determined by outlining the MG lost area. Then its relation to the total area was calculated and expressed as a percentage, that is, the MG dropout (Fig. 1). The presence of MG duct distortion is established if the morphology in the MG is altered by more than 45 degrees (Fig. 1) [23]. Following this definition, distorted MG count was recorded.

\section{CFS}

To enhance the observation of dry spots, the cornea was stained using single-use disposable fluorescein ophthalmic strips (fluorescein sodium, $1 \mathrm{mg}$ ) wetted with nonpreserved buffered saline (sodium chloride, $9 \mathrm{mg} / \mathrm{mL}$ ). The ocular surface was examined with a biomicroscope under both blue-light illumination and a yellow filter after $2 \mathrm{~min}$ of fluorescein sodium staining. CFS was graded from 0 to 12 based on the sum of the fourquadrant scores for the cornea, which were scored individually as 0 (no staining), 1 (mild staining with a few scattered stains), 2 (moderate staining between 1 and 3 ), and 3 (severe staining with confluent stains or corneal filaments) [24].

\section{Statistical analysis}

Statistical analyses were performed with the SPSS 22.0 statistical package. The data discrepancies between the two groups were determined with the Mann-Whitney U tests for, respectively, age, gender, OSDI, TMH, NITBUT, CFS scores, distorted MG count, and MG dropout. The associations of the total MG dropout with age; gender; the duration of CL use; the OSDI; TMH; NITBUT; CFS scores; and distorted MG count were tested using a univariate model and stepwise multiple linear regression models. The F-test with $p$-values set at 0.05 and 0.1 was used to determine each variable's enter and exit criteria for the model, respectively (in collinearity diagnostic tests, all Variance Inflation Factors were $<10$, indicating no multicollinearity). $P$ values lower than 0.05 were considered statistically significant.

\section{Results}

All participants completed all examinations, and none of these subjects experienced any discomfort or pain during the examinations. There were no differences in terms of age $(25.52 \pm 3.20$ vs. $23.35 \pm 3.83$ years old, $P=0.065)$ and the sex ratio (M/F: $23 / 62$ vs. $18 / 45, P=0.622)$ between the CL group and control groups (Table 1). In the CL group, the average duration of CL use was $5.27 \pm$ 4.07 years, ranging from 1 to 18 years.
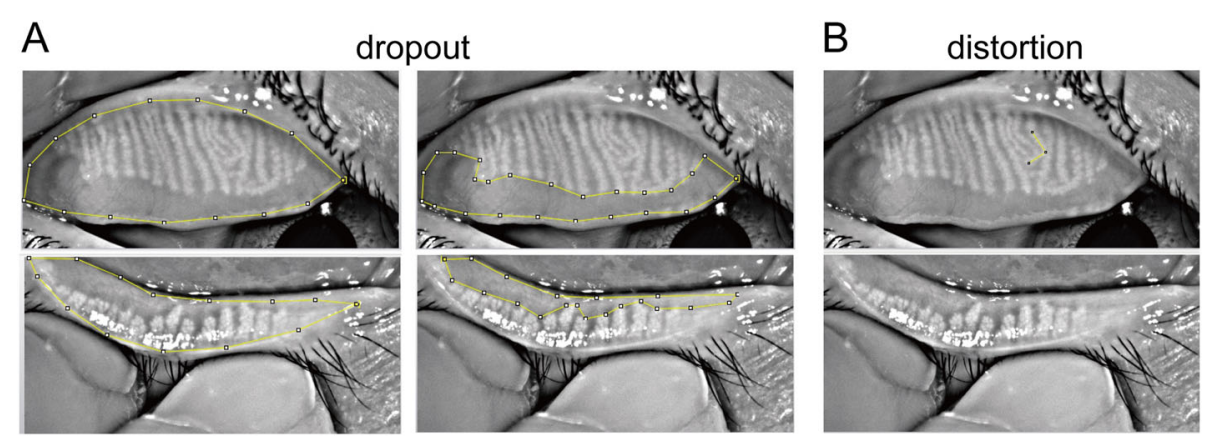

Fig. 1 Method to quantity Meibomian gland changes. a Meibomian gland dropout. Yellow line in the left panel marks the total area (T), and yellow line in the right panel marks out the area with Meibomian gland dropout (D). The dropout ratio is quantified as the ratio between size of $\mathrm{D}$ and T. b. Meibomian gland distortion. Yellow line represents Meibomian gland with distortion. The angle between the line segments is greater than 45 degrees 
Compared to the control group (Table 1), the CL group recorded a higher average OSDI $(17.21 \pm 8.67$ vs. $11.41 \pm 6.00, P<0.001)$, a lower average TMH $(0.21 \pm$ 0.06 vs. $0.24 \pm 0.08 \mathrm{~mm}, P=0.04)$, a lower average NITBUT (6.95 \pm 4.79 vs. $9.26 \pm 2.95 \mathrm{~s}, P<0.001)$, and higher average CFS scores $(2.02 \pm 1.48$ vs. $1.06 \pm 0.88, P<$ 0.001). Compared to the control group (Table 2), the CL group also recorded a higher average total MG dropout ( $28.94 \pm 12.66$ vs. $18.7 \pm 8.78 \%, P<0.001)$, a higher average MG dropout of the upper eyelid $(30.88 \pm 13.34$ vs. $18.42 \pm 8.7 \%, P<0.001)$, a higher average MG dropout of the lower eyelid $(23.26 \pm 17.44$ vs. $16.79 \pm 11.74 \%, P=$ $0.017)$, a larger average total distorted MG count (4.86 \pm 3.44 vs. $2.75 \pm 1.83, P<0.001$ ), a larger average distorted MG count for the upper eyelid $(3.72 \pm 3.09$ vs. $2.03 \pm$ 1.69, $P<0.001$ ), and a larger average total distorted MG count for the lower eyelid $(1.14 \pm 0.97$ vs. $0.71 \pm 0.63$, $P=0.006$ ).

In the univariate analysis (Table 3), a larger total MG dropout was associated with the older age of the subject $(P<0.001)$, larger OSDI $(P=0.006$, Fig. $2 \mathrm{~b})$, larger CFS scores $(P<0.001$, Fig. 2c), and longer duration of CL use $(P<0.001$, Fig. 2a). In the stepwise multivariate analyses, three factors were associated with the total MG dropout $\left(R^{2}=0.356, P<0.001\right.$, Table 3): OSDI $(\beta=0.003)$, CFS $(\beta=0.031)$, and the duration of CL use $(\beta=0.010)$.

In Fig. 3, the $\mathrm{CL}$ group is divided into three small groups according to the duration of CL use (1-2 years, $3-5$ years, and over 5 years) for a more intuitive look at the changes in MG dropout and distorted MG count.

\section{Discussion}

A controversy exists as to whether CL use has an impact on the health of the ocular surface. Chong et al. [25] reported that there was no significant change in the function of the tear film after 7 days of CL use, and Sapkota et al. [7] reported that CL-related discomfort was not associated with the duration of CL use. However, Dogan et al. [26] found that patients with CL-related discomfort usually had a longer CL use history than those without symptoms. Chalmers et al. [27] showed that CL wearers in North America reported longer hours of use with significant aggravation of symptoms of discomfort compared to CL users in the United Kingdom. These previous studies [25-27] regarding CL-related discomfort were all short-term investigations, with the exception of Sapkota's [7]. In the present study, the CL wearers recorded higher OSDI, lower TMH, lower NITBUT, and higher CFS scores. These findings suggest that $\mathrm{CL}$ use gives rise to the increasing severity of CL-related discomfort. Our findings of worsening tear film and quicker evaporation rates in CL wearers agree with the previous studies $[10,17,28]$.

The MGs are large sebaceous glands located in the eyelid. The lipid substances these glands secrete form the lipid layer of the tear film, which acts to prevent tear vaporization and promotes tear film stability. Therefore, disrupting the normal activities of the MGs will alter normal tear physiology through the thinning and breakup of the tear film, interruption of tear film reformation, and disruption of the lipid layer, which results in increased tear film evaporation [29, 30]. Although MG dropout is the most common cause of evaporative dry eye [31], little attention has been paid to a potential role of MG morphologic changes in CL wearers' dry eye symptoms.

Some previous studies have reported that long-term CL use leads to an increase in the MG dropout [32-35]; however, this effect has not been observed in other studies [36-38]. One constraining factor for the application of these findings to other populations is race. The only subjects in Arita's study were Asians, who have an absent or lower crease and more fat in the upper eyelid than Caucasians [39]. The subjects of Other studies were Caucasians [32, 34, 35, 40]. Mechanical trauma from the CLs was determined to cause duct blockage in the MGs [33], and is proportional to the tightness of the eyelid. The results of this study indicate that CL wearers have a higher degree of MG dropout and a larger distorted MG count than non-wearers (Fig. 3). This suggests that CL use is associated with reduced MG morphology, which is consistent with previous reports [33, 35]. The study by Arita et al. [33] investigated MG dropout only, and did not assess distorted MG count. Furthermore, since our study uses a contemporary infrared system, the calculation of MG dropout is more precise.

Aging increases the severity of MG changes in normal individuals [41], which may account for the lower MG dropout rate in the control group. However, Den et al. showed that only a few patients aged less than 50 years had notable abnormalities in the MG [42], but studies

Table 2 Comparison of the MG dropout and distorted MG count for participants from both groups

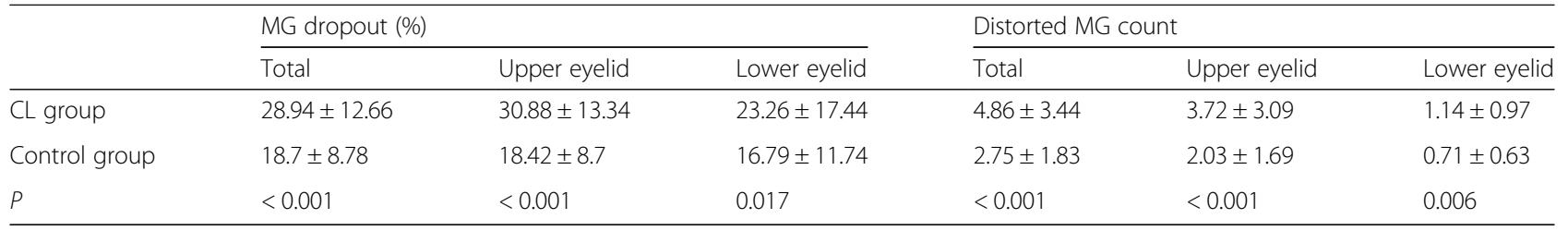

CL contact lens, MGs meibomian glands 
Table 3 Linear regression analyses of the associations of the total MG dropout with age, gender, OSDI, TMH, NIBUT, CFS scores, the number of distort MGs, and the duration of $\mathrm{CL}$ use

\begin{tabular}{|c|c|c|c|c|}
\hline & \multicolumn{2}{|l|}{ Univariate model } & \multicolumn{2}{|l|}{ Multivariate model } \\
\hline & beta $(95 \% \mathrm{Cl})$ & P & beta $(95 \% \mathrm{Cl})$ & P \\
\hline Age (years) & 0.016 (0.008 to 0.024$)$ & $<0.001$ & - & - \\
\hline Gender (male) & $-0.037(-0.099$ to 0.024$)$ & 0.229 & - & - \\
\hline OSDI & 0.004 (0.001 to 0.007 ) & 0.006 & 0.003 (0 to 0.005 ) & 0.043 \\
\hline $\mathrm{TMH}(\mathrm{mm})$ & $0.187(-0.276$ to 0.065$)$ & 0.424 & - & - \\
\hline NIBUT (s) & $-0.003(-0.009$ to 0.003$)$ & 0.311 & - & - \\
\hline CFS scores & 0.038 (0.021 to 0.055$)$ & $<0.001$ & 0.031 (0.016 to 0.047$)$ & $<0.001$ \\
\hline Number of distort MGs & $0.003(-0.005$ to 0.011$)$ & 0.477 & - & - \\
\hline Duration of CL use (years) & 0.014 (0.008 to 0.020$)$ & $<0.001$ & $0.010(0.004$ to 0.016$)$ & 0.001 \\
\hline
\end{tabular}

$C L$ contact lens, OSDI the ocular surface disease index, TMH tear meniscus height, NIBUT noninvasive tear breakup time, CFS corneal fluorescein staining, MGs meibomian glands

also suggest that $\mathrm{CL}$ wear accelerates age-related changes in the MGs [32-35]. Compared with other studies, we used a younger population to explore the effects of CL on the tear film and MG morphology, as this population is known to be more likely to wear CL for a long time. The average age difference between the study and control group was 2.17 years in this study. However, we think the two-year age gap between the 18-30 age range would not make a statistically significant difference based on the above reason. We found that age was significantly associated with the total MG dropout in the univariate linear regression analysis but not in the stepwise multiple linear regression analysis. A significant positive relationship was found between MG dropout and the duration of CL use in both the univariate and multiple linear regression analyses, which is in agreement with previous studies [33]. However, Alghamdi et al. [35] reported that alterations to MG morphology and function accompany $\mathrm{CL}$ wear during the first 2 years of wear, with no further deterioration thereafter. In the early stages of CL use, MG dropout increased significantly, but no further deterioration was found after that [35].

We found a significant positive relationship between MG dropout and OSDI, as well as CFS scores. These results suggest that MG dropout may affect CL-related dryness and maybe one of the potential mechanisms underlying CL-related dry eye. However, Nichols et al. [28] found that the extent of MG dropout was not related to dry eye status in CL wearers. The reason may be that only the MGs in the lower eyelid were analyzed in their study. Both upper and lower eyelids were evaluated in our study, and the total MG dropout was used for the statistical analyses. Similar to the results found in a previous study [33], we found that MG dropout in the upper eyelid $(30.88 \pm 13.34 \%)$ for CL wearers was significantly higher than that of the lower eyelid (23.26 $\pm 17.44 \%)$, and the MG dropout in upper eyelid was closer to the total than that in the lower eyelid. This finding suggests that using the total MG dropout for both eyelids in analyses may be more reliable. This evidence also supports that chronic CLmediated irritation through conjunctiva is a major contributor to MG changes in CL wearers [33, 43] and also that it has a more considerable influence on the MGs of the upper eyelid, especially during blinking.

Complete and functional MGs are essential for normal tear film, particularly in the lipid layer. A healthy lipid layer is integral to tear quality as it provides the tear film with structural integrity [44]. Therefore, damage to any component of

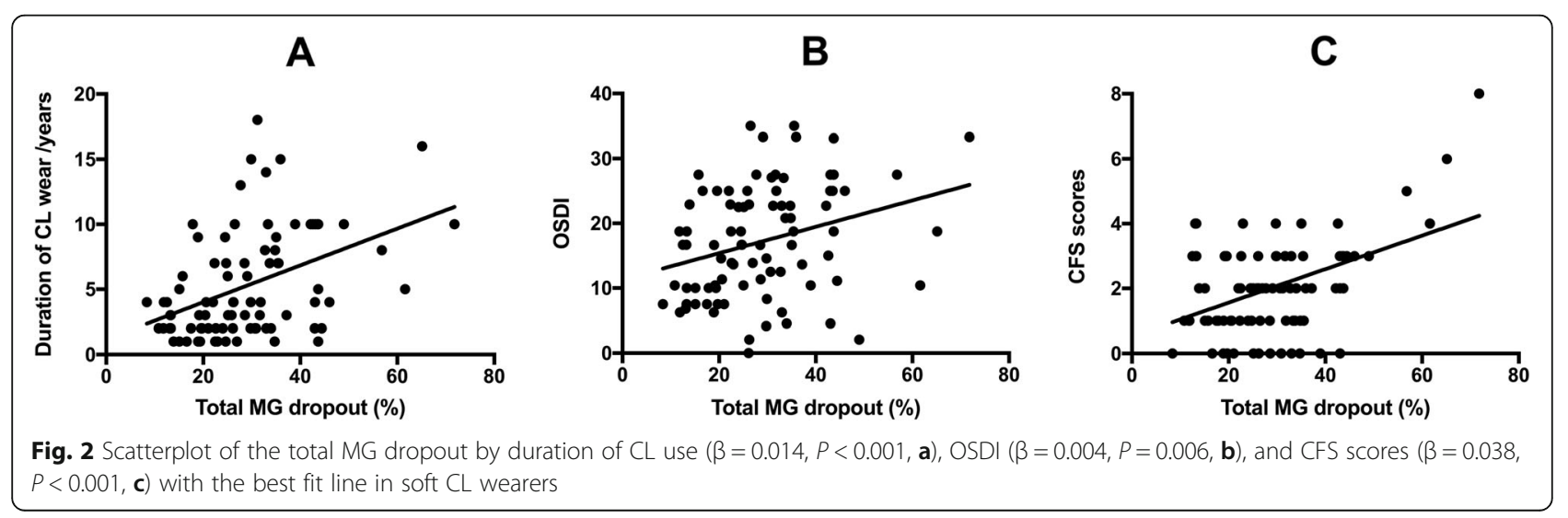


A

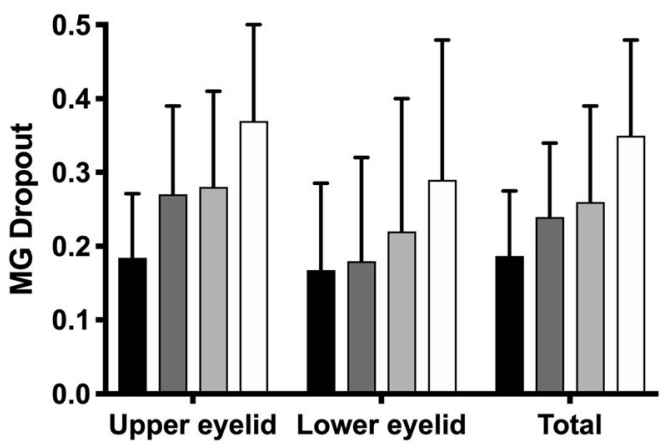

B

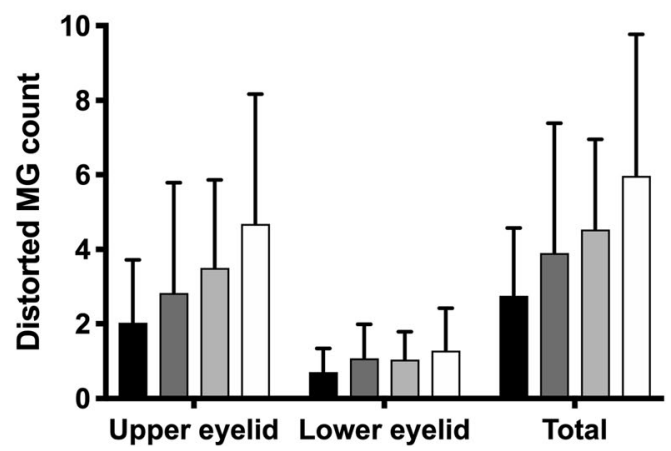

Fig. 3 Comparison of the MG Dropout and Distort MG count for Participants from Both Groups (the CL group were divided into the three small groups by the duration of CL wear: 1-2 years, 3-5 years, and over 5 years)

an MG alters the tear quality, potentially leading to dry eye [16]. In addition, a build-up of meibum in an MG causes ductal dilation, cystic degeneration, loss of the meibocytes, and eventual gland atrophy [16]. Due to the higher degree of MG dropout found in CL wearers, which is supported by the previous $[28,33,45]$ studies, the reduction in meibum volume and quality when secreted onto the ocular surface lead to alterations such as increased evaporation due to the thinning of the lipid layer [16, 31, 46, 47]. Thus, MG dysfunction accompanied by MG dropout presumably plays an important role in the dry eye in CL wearers [48]. However, no significant relationship between MG dropout and TMH $(r=$ $0.088, P=0.424)$ and MG dropout and NIBUT $(r=-0.111$, $P=0.311$ ) was found in this study.

There are several limitations worth noting. First, a large number of studies have shown that the lens material, surface properties, and parameters play a role in CL-related discomfort and that changes in the material, design, and fitting characteristics of CLs may reduce CL discontinuation [13, 4951]. However, this study was limited to the study of a soft CL group versus a non-CL group and did not include additional groups with different CL designs. Second, the refractive error was not adjusted between the $\mathrm{CL}$ and control groups, although the refractive error involved has shown no correlation with CL-related dry eye. Third, the current study did not evaluate the lid margin and MG function. In future studies, a consideration of such as the lipid layer, changes in MG orifices, line of Marx, tarsal plats, tear quantity, tear evaporation, and osmolarity should be considered.

\section{Conclusions}

In conclusion, CL wearers showed higher MG dropout and reduced TMH and NITBUT, which likely contributes to CL-related dry eye symptoms. CL use may lead to a higher MG dropout, and the extent of MG dropout presumably influences the tear film status in $\mathrm{CL}$ wearers.

\section{Abbreviations}

CFS: Corneal fluorescein staining; CL: Contact lens; MGs: Meibomian glands; NIBUT: Noninvasive tear breakup time; OSDI: The ocular surface disease index; $\mathrm{TMH}$ : Tear meniscus height

Acknowledgements

Not applicable.

Authors' contributions

RHW, HN, and SZZ conceived and supervised the experiment. ZZL performed the study. LZ and ZZL collected the data, and TPG analyzed the data. TPG and LZ wrote the manuscript. TPG and LZ contributed equally to this work and were both considered first authors. All authors read and approved the final manuscript.

\section{Funding}

The present study was supported by Grant no. 81770901, from the National Nature Science Foundation of China; by Grant no. 81570834, from the National Nature Science Foundation of China; by Grant no. 17ZXHLSY00070, from the Science Committee Nature Science Foundation of Tianjin, China. and by Grant no. TJLCZDXKT003, from the Tianjin Clinical Key Discipline Project. The funding body had no role in the design or conduct of this research.

Availability of data and materials

The datasets used and/or analyzed during the current study available from the corresponding author on reasonable request.

Ethics approval and consent to participate

This study was approved by the Human Research Ethics Committee of Tianjin Medical University Eye Hospital and was conducted in accordance with the Declaration of Helsinki. Written informed consent was obtained from all enrolled participants.

Consent for publication

We obtained written informed consent for publication from all the participants related to their personal or clinical details and images.

\section{Competing interests}

The authors declare that they have no competing interests.

\section{Author details}

${ }^{1}$ Tianjin Key Laboratory of Retinal Functions and Disease, Tianjin 300384, China. ${ }^{2}$ Eye Institute and School of Optometry, Tianjin 300384, China. ${ }^{3}$ Tianjin Medical University Eye Hospital, Tianjin 300384, China. 
Received: 7 May 2019 Accepted: 23 January 2020

Published online: 04 March 2020

\section{References}

1. Nichols JJ, Willcox MD, Bron AJ, et al. The TFOS International Workshop on Contact Lens Discomfort: executive summary. Invest Ophthalmol Vis Sci. 2013;54(11):TFOS7-TFOS13.

2. Nichols JJ. CONTACT LENSES 2017. Contact Lens Spectrum; 2018.

3. Guillon M, Maissa C. Dry eye symptomatology of soft contact lens wearers and nonwearers. Optom Vis Sci. 2005;82(9):829-34.

4. Nichols JJ, Ziegler C, Mitchell GL, et al. Self-reported dry eye disease across refractive modalities. Invest Ophthalmol Vis Sci. 2005;46(6):1911-4.

5. Li W, Sun X, Wang Z, et al. A survey of contact lens-related complications in a tertiary hospital in China. Cont Lens Anterior Eye. 2018;41(2):201-4.

6. White CJ, Dipasquale SA, Byrne ME. Controlled release of multiple therapeutics from silicone hydrogel contact lenses. Optom Vis Sci. 2016; 93(4):377-86.

7. Sapkota K, Martin R, Franco S, et al. Common symptoms of Nepalese soft contact lens wearers: a pilot study. J Opt. 2015;8(3):200-5.

8. Pili K, Kastelan S, Karabatic M, et al. Dry eye in contact lens wearers as a growing public health problem. Psychiatr Danub. 2014;26(Suppl 3):528-32.

9. Richdale $K$, Sinnott LT, Skadahl E, et al. Frequency of and factors associated with contact lens dissatisfaction and discontinuation. Cornea. 2007;26(2): $168-74$.

10. Chalmers RL, Begley CG. Dryness symptoms among an unselected clinical population with and without contact lens wear. Cont Lens Anterior Eye. 2006;29(1):25-30.

11. Downie LE, Craig JP. Tear film evaluation and management in soft contact lens wear: a systematic approach. Clin Exp Optom. 2017;100(5):438-58.

12. Papas E, Tilia D, Mcnally J, et al. Ocular discomfort responses after short periods of contact lens wear. Optom Vis Sci. 2015;92(6):665-70.

13. Sapkota K, Franco S, Sampaio P, et al. Effect of three months of soft contact lens wear on conjunctival cytology. Clin Exp Optom. 2016;99(4):336-41.

14. Best N, Drury L, Wolffsohn JS. Predicting success with silicone-hydrogel contact lenses in new wearers. Cont Lens Anterior Eye. 2013;36(5):232-7.

15. Sengor T, Aydin Kurna S, Ozbay N, et al. Contact lens-related dry eye and ocular surface changes with mapping technique in long-term soft silicone hydrogel contact lens wearers. Eur J Ophthalmol. 2012;22(Suppl 7):S17-23.

16. Nichols KK, Foulks GN, Bron AJ, et al. The international workshop on meibomian gland dysfunction: executive summary. Invest Ophthalmol Vis Sci. 2011:52(4):1922-9.

17. Faber E, Golding TR, Lowe R, et al. Effect of hydrogel lens wear on tear film stability. Optom Vis Sci. 1991;68(5):380-4.

18. Schiffman RM, Christianson MD, Jacobsen $\mathrm{G}$, et al. Reliability and validity of the ocular surface disease index. Arch Ophthalmol. 2000;118(5):615-21.

19. Koh S, Ikeda C, Watanabe $\mathrm{S}$, et al. Effect of non-invasive tear stability assessment on tear meniscus height. Acta Ophthalmol. 2015;93(2):e135-9.

20. Hong J, Sun $X$, Wei $A$, et al. Assessment of tear film stability in dry eye with a newly developed keratograph. Cornea. 2013;32(5):716-21.

21. Srinivasan $S$, Menzies $K$, Sorbara $L$, et al. Infrared imaging of meibomian gland structure using a novel keratograph. Optom Vis Sci. 2012;89(5): 788-94.

22. Satjawatcharaphong P, Ge S, Lin MC. Clinical outcomes associated with thermal pulsation system treatment. Optom Vis Sci. 2015;92(9):e334-41.

23. Arita $\mathrm{R}$, Itoh $\mathrm{K}$, Maeda $\mathrm{S}$, et al. Meibomian gland duct distortion in patients with perennial allergic conjunctivitis. Cornea. 2010;29(8):858-60.

24. Afonso AA, Monroy D, Stern ME, et al. Correlation of tear fluorescein clearance and Schirmer test scores with ocular irritation symptoms. Ophthalmology. 1999;106(4):803-10.

25. Chong $P Q$, Yeo $S$, Too $C L$, et al. Effects of wearing a daily disposable lens on tear film: a randomised controlled trial. Clin Exp Optom. 2016;99(3):241-7.

26. Dogan AS, Gurdal C, Arslan N. Corneal confocal microscopy and dry eye findings in contact lens discomfort patients. Cont Lens Anterior Eye. 2018; 41(1):101-4

27. Chalmers RL, Young G, Kern J, et al. Soft contact Lens-related symptoms in North America and the United Kingdom. Optom Vis Sci. 2016:93(8):836-47.

28. Nichols JJ, Sinnott LT. Tear film, contact lens, and patient-related factors associated with contact lens-related dry eye. Invest Ophthalmol Vis Sci. 2006;47(4):1319-28.

29. Knop E, Knop N, Millar T, et al. The international workshop on Meibomian gland dysfunction: report of the subcommittee on anatomy, physiology, and pathophysiology of the Meibomian gland. Invest Ophthalmol Vis Sci. 2011:52(4):1938-78.

30. Mcmonnies CW. Psychological and other mechanisms for end-of-day soft lens symptoms. Optom Vis Sci. 2013;90(6):e175-81.

31. Bron AJ, Tiffany JM. The contribution of meibomian disease to dry eye. Ocul Surf. 2004;2(2):149-65.

32. Machalinska A, Zakrzewska A, Adamek B, et al. Comparison of morphological and functional Meibomian gland characteristics between daily contact Lens wearers and nonwearers. Cornea. 2015;34(9):1098-104.

33. Arita $\mathrm{R}$, Itoh $\mathrm{K}$, Inoue $\mathrm{K}$, et al. Contact lens wear is associated with decrease of meibomian glands. Ophthalmology. 2009:116(3):379-84.

34. Villani E, Ceresara G, Beretta S, et al. In vivo confocal microscopy of meibomian glands in contact lens wearers. Invest Ophthalmol Vis Sci. 2011;52(8):5215-9.

35. Alghamdi WM, Markoulli M, Holden BA, et al. Impact of duration of contact lens wear on the structure and function of the meibomian glands. Ophthalmic Physiol Opt. 2016;36(2):120-31.

36. Marren SE. Contact lens wear, use of eye cosmetics, and Meibomian gland dysfunction. Optom Vis Sci. 1994;71(1):60-2

37. Hom MM, Martinson JR, Knapp LL, et al. Prevalence of Meibomian gland dysfunction. Optom Vis Sci. 1990;67(9):710-2.

38. Ong BL. Relation between contact lens wear and Meibomian gland dysfunction. Optom Vis Sci. 1996:73(3):208-10.

39. Jeong S, Lemke BN, Dortzbach RK, et al. The Asian upper eyelid: an anatomical study with comparison to the Caucasian eyelid. Arch Ophthalmol (Chicago, III: 1960). 1999;117(7):907-12.

40. Schaumberg DA, Nichols JJ, Papas EB, et al. The international workshop on meibomian gland dysfunction: report of the subcommittee on the epidemiology of, and associated risk factors for, MGD. Invest Ophthalmol Vis Sci. 2011;52(4):1994-2005.

41. Arita R, Itoh $\mathrm{K}$, Inoue $\mathrm{K}$, et al. Noncontact infrared meibography to document age-related changes of the meibomian glands in a normal population. Ophthalmology. 2008;115(5):911-5.

42. Den S, Shimizu K, Ikeda T, et al. Association between meibomian gland changes and aging, sex, or tear function. Cornea. 2006;25(6):651-5.

43. Ong BL, Larke JR. Meibomian gland dysfunction: some clinical, biochemical and physical observations. Ophthalmic Physiol Opt. 1990;10(2):144-8.

44. Millar TJ, Schuett BS. The real reason for having a meibomian lipid layer covering the outer surface of the tear film - a review. Exp Eye Res. 2015;137:125-38.

45. Arita R, Itoh $\mathrm{K}$, Maeda S, et al. Proposed diagnostic criteria for obstructive meibomian gland dysfunction. Ophthalmology. 2009;116(11):2058-2063.e2051.

46. Vishnubhatla S, Borchman D, Foulks GN. Contact lenses and the rate of evaporation measured in vitro; the influence of wear, squalene and wax. Cont Lens Anterior Eye. 2012;35(6):277-81.

47. Rohit A, Willcox M, Stapleton F. Tear lipid layer and contact lens comfort: a review. Eye Contact Lens. 2013;39(3):247-53.

48. Khaireddin R, Hueber A. Eyelid hygiene for contact lens wearers with blepharitis. Comparative investigation of treatment with baby shampoo versus phospholipid solution. Ophthalmologe. 2013;110(2):146-53.

49. Vidal-Rohr M, Wolffsohn JS, Davies LN, et al. Effect of contact lens surface properties on comfort, tear stability and ocular physiology. Cont Lens Anterior Eye. 2018;41(1):117-21.

50. Michaud L, Forcier P. Comparing two different daily disposable lenses for improving discomfort related to contact lens wear. Cont Lens Anterior Eye. 2016:39(3):203-9.

51. Truong TN, Graham AD, Lin MC. Factors in contact lens symptoms: evidence from a multistudy database. Optom Vis Sci. 2014;91(2):133-41.

\section{Publisher's Note}

Springer Nature remains neutral with regard to jurisdictional claims in published maps and institutional affiliations. 